\begin{tabular}{|c|l|}
\hline Title & Phenotypic grouping of 141 BmNPV s lacking viral gene sequences \\
\hline Author(s) & Ono, Chikako; Kamagata, Takanori; Taka, Hitomi; Sahara, Ken; A sano, Shin-ichiro; Bando, Hisanori \\
\hline Citation & $\begin{array}{l}\text { Virus Research, 165(2), 197-206 } \\
\text { https://doi.org/10.1016j.virusres.2012.02.016 }\end{array}$ \\
\hline Issue Date & 2012-05 \\
\hline Doc URL & http://hdl.handle.net/2115/49265 \\
\hline Type & article (author version) \\
\hline File Information & VR165-2_197-206.pdf \\
\hline
\end{tabular}

Instructions for use 


\section{Phenotypic grouping of 141 BmNPVs lacking viral gene sequences}

Chikako Ono, Takanori Kamagata, Hitomi Taka, Ken Sahara, Shin-ichiro Asano and Hisanori Bando*

Laboratory of Applied Molecular Entomology, Division of Applied Bioscience, Graduate School of Agriculture, Hokkaido University, Sapporo 060-8589, Japan

Running title: Phenotypic grouping of 141 knockout BmNPVs

* Corresponding author: Prof. Hisanori Bando

Mailing address: Laboratory of Applied Molecular Entomology, Division of Applied

Bioscience, Graduate School of Agriculture, Hokkaido University, Sapporo 060-8589, Hokkaido, Japan. Phone \& Fax : +81-11-706-3348.

E-mail : hban@abs.agr.hokudai.ac.jp 


\begin{abstract}
We constructed a series of gene knockout BmNPVs (KOVs) for each of 141 genes using the BmNPV T3 bacmid system and lambda red recombination system. In a subsequent analysis of the properties needed for infection using a marker gene, egfp (enhanced green fluorescent protein gene), inserted into the polyhedrin locus, the knockout viruses (KOVs) were subdivided into four phenotypic types, A to D. Type-A (86 KOVs) showed the ability to expand infections equivalent to the control while type-B (8 KOVs) spread infections more slowly. Type-C (37 KOVs) expressed egfp in transfected-BmN cells but the production of infectious viruses was not observed. Type-D (10 KOVs) showed no ability to express egfp even in the transfection experiments. KOVs lacking genes (pkip (Bm15), gp41 (Bm66), bro-d (Bm131), Bm20, 48, 65, 91, 93, or 101) previously identified as being essential, were placed in the viable type-A and B categories.
\end{abstract}

Keywords: BmNPV, bacmid, lambda red recombination system, gene knockout

Highlights: > We constructed a series of gene knockout BmNPVs (KOVs) for each of 141 genes using BmNPV T3 bacmid system. > KOVs were subdivided into four phenotypic types by polyhedrin promoter-driven EGFP expression and infectious behavior. > The catalogue of BmNPV genes will be a helpful reference in the functional analysis of viral genes. 


\section{Introduction}

Nucleopolyhedrovirus (NPV) is one of the largest DNA viruses. Its genome contains

over 100 genes, which are expressed in a stage-dependent manner; immediate-early, delayed-early, late and very-late (Friesen and Miller, 1986). Genes of NPV are involved in transcriptional regulation (Guarino and Summers, 1986; Yoo and Guarino, 1994), viral RNA polymerase components (Guarino et al., 1998), nucleocapsid formation (Thiem and Miller, 1989; Vanarsdall et al., 2006), host gene regulation (Nobiron et al., 2003) and so on. About half of them are predicted to be essential for viral propagation through gene expression, DNA replication and virion components (Rohrmann, 2011). On the other hand, there are some accessory genes or non-essential genes that remain in the viral genome during passage. These observations suggested that each viral gene would work to the advantage at least in certain situations through interaction with other viral/host genes.

The function of baculoviral genes in replication has been studied vigorously by using gene knock-out (KO) technology in baculoviruses such as Autographa californica multiple NPV (AcMNPV) and Bombyx mori NPV (BmNPV) (Rohrman et al., 2011), however, the function of more than one-third of viral genes are still unknown. In addition, KO viruses have been generated based on different genetic backbones using various methods, that is; different species (AcMNPV or BmNPV), strains and methods for mutation (temperature-sensitive mutant, deletion or insertion into a wild-type baculoviral genome or bacmid). In the 1980 s, KO viruses were obtained by conventional homologous recombination in insect cells, sometimes making it difficult to distinguish the negative results caused by a failure of the recombination from those caused by the knock-out of an essential gene. This problem was solved when the bacmid system was established in 
AcMNPV (Luckow et al., 1993) since the viral (bacmid) DNAs could be amplified in $E$. coli even if they lacked essential genes for replication in the host insect cells. Now this technique is available for other baculoviruses such as BmNPV (Motohashi et al., 2005) and Helicoverpa armigera SNPV (HearNPV) (Wang et al., 2003).

In general, early genes are transcribed by host RNA polymerase II and mainly involved in regulating replication as trans-regulators, however, late genes are transcribed by viral-derived RNA polymerase and involved in forming viral structure as capsid proteins. In addition, there have been reports that the expression of host genes was not only down-regulated (i.e. shut off), but up-regulated during baculovirus infections (Nobiron et al., 2003; Sagisaka et al., 2010.). These observations suggested that the baculovirus controlled the host cell mechanisms to produce progeny viruses using host factors and machinery through a complex gene regulatory mechanism among not only viral genes but host genes. To understand the mechanisms of viral replication, insight into the functions of baculoviral genes and host genes responding to viral infections is essential. Among lepidopteran insects, which include many hosts of baculoviruses, Bombyx mori is well-studied physiologically, biochemically and molecular biologically. It is worth noting that the genome has been sequenced through international collaboration between Japan and China (International Silkworm Genome Consortium, 2008), suggesting that the informational environment for analyzing the interaction between host and virus is being put into place in the BmNPV-silkworm infection system.

To establish a platform for the comprehensive analysis of the BmNPV gene network and/or interaction between viral and host genes, we constructed a series of gene knock-out BmNPVs (KOVs) for each of 135 genes (Gomi et al., 1999) and another 6 ORFs (Katsuma et al., 2011) using the BmNPV T3 bacmid system (Ono et al., 2007) and lambda red 
recombination system (Datsenko and Wanner, 2000). Subsequently the growth properties of KOVs in BmN cells, and the gene knockout-specific effects on the production of infectious progeny and polyhedrin gene expression were analyzed using a marker (GFP) controlled by the polh promoter. 


\section{Materials and methods}

\section{1. Cells, bacmids and transfection.}

$\mathrm{BmN}$ cells were maintained in TC-100 medium (Applichem) containing 10\% FBS at $26^{\circ} \mathrm{C}$. Transfection of $\mathrm{BmN}$ cells with bacmid DNAs was performed by lipofection using FuGENE HD Transfection Reagent (Promega) according to the manufacturer's instructions.

Escherichia coli $(E$ coli.) strains BW25113 (containing pKD46 encoding the genes for the lambda red recombination system) and BW25141 (pKD3 encoding a chloramphenicol acetyltransferase gene (cat)) (Datsenko and Wanner, 2000) were provided by the E. coli Genetic Stock Center (Yale University, USA).

\section{2. Generation of knockout BmNPV bacmids expressing EGFP.}

The BmNPV bacmid system (T3 strain, [Ono et al., 2007]) was used to generate knockout viruses with the lambda red recombination system (Datsenko and Wanner, 2000).

We first generated a transfer vector for the Golyhedron (polh) locus of the bacmids. The enhanced green fluorescent protein (EGFP) coding sequence (egfp) was excised from pEGFP-1 (Clontech) by digestion with BamH I and Not I and ligated into the BamH I-Not I site of pFastBac1 (Invitrogen), yielding pFastBac-GFP. The BmNPV T3 bacmid expressing EGFP under the control of the polh promoter was produced by transposition in E coli. (BmT3DH10Bac; containing the BmNPV genome bacmid and a helper plasmid pMON7124 encoding a transposase) with pFastBac-GFP. The bacmid DNA carrying egfp was isolated from a kanamycin-, gentamycin-resistant and lacZ-negative colony, and designated Bmbac ${ }^{+ \text {egfp }}$ (BmGFP) as described elsewhere (Ono et al., 2007).

For knocking out each gene, we used the lambda red recombination system (Datsenko 
and Wanner, 2000). KOVs were generated by homologous recombination in E. coli containing pKD46 as a helper plasmid encoding a lambda red recombinase to replace each target gene with a chloramphenicol acetyltransferase (cat) gene from pKD3 for antibiotic selection. Briefly, DH10B cells (Invitrogen) containing pKD46 were transfected with BmGFP DNA by electroporation using a GenePulser (Biorad) $(25 \mu \mathrm{F}, 2.5 \mathrm{kV}, 200 \Omega)$ and designated BmT3DH10B ${ }^{+ \text {egfp }}$-pKD46. Then, the cat gene sequences wedged between the 5' non-coding regions (50 nucleotides (nts)) and the $3^{\prime}$ non-coding regions (50 nts) of the BmNPV target genes were amplified by PCR with the primer sets (Table. 1) using pKD3 as a template. Primers for knocking out a gene were designed not to prevent the expression of adjacent genes, that is, promoter motifs (CAGT, TATA, and TAAG) and at least $30 \mathrm{nts}$ upstream from the ATG translation start codon of the adjacent genes remained. The length of each deleted region was from $34(\Delta B m 95 a)$ to 3605 nts ( $\triangle$ dnahel $(B m 78)$ ). After Dpn I treatment to digest pKD3, PCR products (approximately $1 \mathrm{kbp},>500 \mathrm{ng}$ ) were purified using Wizard SV Gel and a PCR Clean-Up System (Promega) and transformed into BmT3DH10B ${ }^{+ \text {egfp }}$-pKD46 by electroporation as above. Then, cells were incubated with $0.01 \%$ Arabinose SOC broth for $4-6 \mathrm{~h}$ at $37^{\circ} \mathrm{C}$. Each gene-knockout BmNPV bacmid was obtained from an chloramphenicol- and kanamycin-resistant colony, followed by verification of the absence of the viral gene ORF by PCR with gene-specific primer sets targeting the sequence (about $300 \mathrm{nts}$ ) inside of each gene, which were designed for a baculovirus DNA microarray (Yamagishi et al., 2003). On the other hand, the existence of the cat sequence in each KOV was confirmed by PCR with primers to amplify the sequence surrounding the 5'-terminus (cat-up: 5'-gaatcagctccagctacac-3' and the gene-specific primers) or the 3'-terminus (cat-down: 5'-ctaaggaggatattcatatg-3' and the gene-specific primers) of the cat sequence. After incubation at $37^{\circ} \mathrm{C}$ for 6 hours to remove the helper plasmid pKD46 from bacteria, Ampicillin-sensitive colonies were selected. Bacmid DNAs 
were purified from 50-ml LB cultures using a Qiagen midi-plasmid kit (Qiagen) and each concentration was determined by NanoDrop2000 (Thermo scientific).

\section{3. KOV transfection and infection assay}

BmN cells were washed with serum-free TC-100 medium and seeded into 96 well plates $\left(5 \times 10^{4}\right.$ cells/well). The cells were transfected with each bacmid $(0.25 \mu \mathrm{g})$ as described above and subjected to fluorescence microscopic observation. The fluorescence intensity of EGFP was monitored by infinite M200PRO (Tecan). At 4 days post transfection (d.p.t.), the supernatant $(10 \mu \mathrm{l})$ was collected and added to freshly seeded BmN cells. The culture was continued and the fluorescence intensity of these cells was measured daily as above. 


\section{Results}

\section{1. Generation of BmNPV knockout bacmids.}

BmNPV has about 140 genes, however, most of their functions were remain unknown. To investigate their role in the viral replication cycle, we generated 141 genes-knockout viruses. The absence of the target gene in each KOV was verified by PCR. PCR targeting a sequence of $0.3 \mathrm{kbp}$ inside each deleted ORF was carried out for the knockout bacmids, resulting in negative for each ORF (Fig. 1(b): results for $\Delta B m 20, \Delta B m 48, \Delta B m 91, \Delta B m 93$ and $\triangle B m 101$ are shown as examples). On the other hand, PCR with the primer sets targeting the 5'-terminal region (Fig. 1(c)) or the 3'-terminal region (Fig. 1(d)) of the cat sequence resulted in the amplification of DNA fragments of expected size for the 5'-terminal region (1500 nucleotides (nts) for $\Delta B m 20,800 \mathrm{nts}$ for $\Delta B m 48,1200 \mathrm{nts}$ for $\Delta B m 91,2000 \mathrm{nts}$ for $\Delta B m 93,500 \mathrm{nts}$ for $\Delta B m 101)$ or the 3'-terminal region (650 nts for $\Delta B m 20,300 \mathrm{nts}$ for $\Delta B m 48,750 \mathrm{nts}$ for $\Delta B m 91,450 \mathrm{nts}$ for $\Delta B m 93,700 \mathrm{nts}$ for $\Delta B m 101)$. We confirmed the knocked out region of other KOVs in the same way (data not shown). The KOV $(\triangle B m 95 a)$ failed possibly because the sequence to be deleted for $B m 95 a$ was very small (34 nts). Therefore, we analyzed a KOV lacking both Bm95a and Bm96 $(\Delta B m 95 a-96)$.

\section{2. The growth properties of each KOV.}

To define each KOV phenotype, we introduced the bacmids into BmN cells and analyzed the EGFP expression every 24 hours. In addition, at 96 hours post transfection (h.p.t.), the culture medium was collected and inoculated into the new cell layer in order to determine whether the infectious virion was produced or not. Because EGFP was driven by the polh 
promoter in each KOV-transfected cell, we observed the infection and expression of polh based on the intensity of EGFP in the cells.

The KOV (bacmid)s were roughly subdivided into four phenotypes (A to D); type-A and -B KOVs produced infectious viruses but type-C and -D did not (Fig. 2). At 48 h.p.t., EGFP expression was detected in the BmN cells transfected with 131 KOV DNAs (Fig. 2) in addition to the cells transfected with BmGFP DNA, a control (polh replaced with egfp) bacmid. At 96 h.p.t., the expansion of the cells expressing EGFP was observed in the cells transfected with $86 \mathrm{KOV}$ DNAs (see Fig. 2, photograph of $\triangle B m 101$ bacmid-transfected $\mathrm{BmN}$ cells; type-A KOV). The ability to expand an infection was confirmed by experiments using the culture medium of the cells transfected with the $86 \mathrm{KOVs}$ as inoculums. Many type-A KOVs were able to spread infections (=cell-to-cell infection) as fast as BmGFP (a control), however, $8 \mathrm{KOVs}(\Delta B m 5, \Delta l e f-6$ (Bm19), $\Delta$ ubiquitin (Bm26), $\Delta l e f-12$ (Bm32), $\Delta B m 36, \Delta o d v-e 66$ (Bm37), $\Delta B m 56, \Delta i a p 2(B m 58))$ showed less activity for EGFP expression ( $<30 \%$, compared to BmGFP at 96 h.p.t.). On the other hand, the EGFP levels in the transfected cells were almost the same as in the BmGFP-transfected cells but the infection did not spread to all cells in the case of the $8 \mathrm{KOVs}(<40 \%$, compared to BmGFP at 96 h.p.t.; see Fig. 2, photograph of Apkip (Bm15) bacmid-transfected $\mathrm{BmN}$ cells; type-B KOV). In addition, in a second infection in newly seeded cells, the fluorescence intensity of EGFP was low. Then, for $37 \mathrm{KOVs}$, EGFP expression was verified but no cell-to-cell infection was observed at least not at 96 h.p.t. (see Fig. 2, photograph of $\Delta d b p(B m 16)$ bacmid-transfected BmN cells; type-C KOV). In the subsequent infection experiment, we couldn't detect any EGFP in the cells (data not shown). Finally, there were $10 \mathrm{KOVs}$ for which EGFP expression wasn't observed not only in transfected BmN cells, but also in infected cells (see Fig. 2, photograph of $\Delta l e f-9$ (Bm50) bacmid-transfected BmN cells; type-D KOV). 


\section{Discussion}

We generated KOVs for 141 genes of BmNPV using a bacmid system and the lambda red recombination system and characterized their phenotype (Table 2). Forty-five of these KOVs have not been reported for not only BmNPV but also AcMNPV previously (Table.2).

Type-A KOVs ( $\mathrm{n}=86)$ were able to spread infections as fast as a control BmGFP, though much less GFP than BmGFP ( $<30 \%$ at 96 h.p.t.) was expressed for eight KOVs $(\Delta B m 5$,

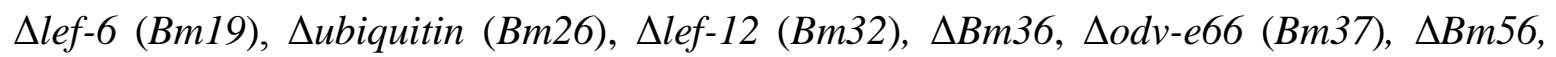
$\Delta$ iap2 (Bm58)). It was previously reported that the deletion of Bm56 in BmNPV affected the occlusion body's morphogenesis (Xu et al., 2008). In addition, the deletion of lef-6 or lef-12 in AcMNPV resulted in a decrease in the expression of late and very-late genes including polh (Lin and Blissard, 2002; Guarino et al., 2002). These observations were consistent with our results. On the other hand, though AcMNPV lef-7 was also identified as late expression factor and reported to stimulate DNA replication (Morris et al., 1994) and polyhedra formation in SF21 cells (Chen and Thiem, 1997), we observed no significant differences in viral production and Polh expression between lef-7 KOV and the control bacmid BmGFP

Type-B KOVs ( $\mathrm{n}=8)$ were characterized by a markedly slow spreading of the infection probably because of the low production of infectious viruses and/or low infectivity of the progeny viruses. Viruses lacking pkip (Bm15) (temperature-sensitive mutant, AcMNPV L1 [McLachlin et al., 1998]), Bm65 (failed in homologous recombination, BmNPV T3

[Rohrmann, 2011]), gp41 (Bm66) (temperature-sensitive mutant, AcMNPV L1 [Olszewski 
and Miller, 1997]) and bro-d (Bm131) (homologous recombination failed, BmNPV T3 [Kang et al., 1999]) were also viable (type-B).

pkip codes for a protein kinase interacting protein (PKIP) which stimulates a virus-encoded protein kinase (Pk1) activity in vitro (McLachlin et al., 1998) which may explain our observations $(\triangle \mathrm{Pk}-1(B m 3)$ was grouped into type-C and pkip (Bm15) was type-B)

The $g p 41(\mathrm{Bm} 66)$ product is not a component of budded viruses (BVs) but is required for the egress of nucleocapsids from the nucleus in the process of BV synthesis (Whitford and Faulkner, 1992). Olszewski and Miller (1997) reported that a ts mutant of gp4l was able to form occlusion bodies in Sf21 cells, but couldn't produce infectious progeny. These reports suggested $g p 41$ to play important roles in the process of virus production that is consistent with our observations though gp4l-KO BmNPV was not like complete defective in the productivity of infectious progeny.

The functions of Bm65 and bro-d in virion production are still unclear. The orf79 of AcMNPV, a homolog of the Bm65 of BmNPV, was found to code for a protein associating with ODV and predicted to be a member of the UvrC superfamily of endonucleases involved in DNA repair (Aravind et al., 1999). The baculovirus repeated ORF (bro) family is unique and involved in nuclear export, shuttling mRNA between the nucleus and cytoplasm (Kang et al., 2006). BmNPV contains five bro genes with bro-d thought to play an important role in viral infection because of a failure to isolate a bro- $d$ deletion mutant. The lack of such functions might explain the defective (type-B) infection of Bm65 KOV or bro-d $\mathrm{KOV}(\triangle B m 65$ or $\Delta b r o-d)$ observed in this study but more experiments are required. 
Type-C KOVs $(n=37)$ expressed GFP in the $\mathrm{BmN}$ cells transfected with the bacmid DNAs but no spreading of the infection was observed. Most of the genes knocked out in type-C KOVs code for virion structural proteins (shown in Table 2, (Braunagel et al., 2003; Wang et al., 2010). In type-C KOV-transfected cells, very-late gene expression was observed but cell-to-cell infection (the production of infectious progeny) was not. These observations suggested the genes to play important roles in the morphogenesis of BmNPV. Interestingly, lef-1 (Bm6), lef-2 (Bm135) and dnapol (Bm53) that were required for transient DNA replication (Kool et al., 1994.; Lu and Miller, 1995) were not necessary for polh expression (type-C KOVs), indicating that DNA replication and very-late gene expression were regulated independently. In addition, we observed the expression of GFP in the cells transfected with $d b p(B m 16) \mathrm{KOV}(\Delta d b p)$. The $d b p$ gene is required for the maturation of the virogenic stroma (Mikhailov et al., 2008; Nagamine et al., 2006; Vanarsdall et al., 2007), suggesting that the very-late gene expression was independent of the formation of the virogenic stroma.

In the cells transfected with type-D bacmid DNAs $(n=10)$, neither the expression of GFP nor the production of infectious viruses was detected. The 10 viral genes lacking in type-D KOVs were essential not only for viral replication, but for polh expression. This observation is consistent with reports that they were essential for DNA replication or late gene expression as components of viral RNA polymerase (Kool et al., 1994; Lu et al., 1995; Guarino et al., 1998).

Some 57 essential genes have been recognized in BmNPV (Gomi et al., 1997; Kang et al., 1999; Rohrmann, 2011), mainly based on the results of homologous recombination experiments and information on the function of homologues in AcMNPV. We here adopted 
the bacmid system which allows the construction of KOV DNA lacking even essential genes to reevaluate the necessity of BmNPV genes in viral reproduction.

Our results indicated that viruses lacking one of 9 BmNPV genes $(B m 20,48,65,91,93$, 101, pkip (Bm15), gp41 (Bm66) and bro-d (Bm131)), which were originally identified as essential, generated infectious progeny (type-A, B). The discrepancy could be due to difficulties in generating these insertion/deletion mutants in the homologous recombination system.

Bm20, 48, 91, 93 and 101 were originally identified as essential because no insertion/deletion mutants of these genes could be isolated from BmNPV strain T3 (Rohrmann, 2011). However, in this study using a bacmid system, the viruses lacking these genes produced infectious viruses as well as a control BmGFP in BmN cells (type-A). $B m 48$ is the homologue of AcMNPV orf60, which is a homologue of ChaB, a putative membrane ion antiporter regulator from Escherichia coli. The ChaB homologues identified in Spodoptera litura (Spli) NPV were suggested to code for DNA-binding proteins (Li et al., 2006). Furthermore, a recent study of the homolog in HearNPV, Ha51, reported that was not essential for virus production (Zheng et al., 2011), and our result in BmNPV was consistent with thier report. The homolog of Bm91 (in Antheraea pernyi NPV [Shi et al., 2007]) was an ODV-associated protein and that of Bm101 (in AcMNPV [Wang et al., 2010]), a BV-associated protein. The results of this study suggested that the functions of these genes were not required for BmNPV reproduction in $\mathrm{BmN}$ cells though these genes are conserved among Group I NPV (Lepidoptera NPV) (Jehle et al., 2006). However, our results do not exclude the possibility that these genes have important roles in the viral infection cycle in vivo. 
In this study, we generated KOVs for $141 \mathrm{BmNPV}$ genes and sub-divided the KOVs into four phenotypic groups. The catalogue of BmNPV genes and their relationship to a criterion for their replication presented here will be a helpful reference in the functional analysis of viral genes though other experiments are required for evaluating the knockouts that showed an effect in detail. In addition, the series of sequence deletion mutants in BmNPV constructed here with the lambda red recombination system will provide a useful tool in systems biology for identifying the interaction among viral/host genes.

\section{Acknowledgments}

This work was supported in part by the Research Fellowships of the Japan Society for the Promotion of Science (JSPS) for Young Scientists (to C. O.) and a Grant-in-Aid for Scientific Research from the Ministry of Education, Science, and Culture of Japan (MEXT Grant, Grant No. 22248003). 


\section{FIGURE LEGENDS}

Table 1. Oligonucleotide primers used in the PCR to generate knockout bacmids.

To construct KOVs using the lambda red recombination system (Datsenko and Wanner, 2000), the cat genes with sequences for gene-targeting recombination were synthesized by PCR with each primer set. Oligonucleotide primer sequences, the target gene name and the

orf number of BmNPV are shown. 5' primer; cat gene 5'-terminal sequence in pKD3 (gtgtaggctggagctgcttc) combined with the upstream sequences of target genes. 3' primer: cat gene 5'-terminal sequence in pKD3 (catatgaatatcctccttag) combined with the downstream sequences of target genes.

\section{Fig. 1. Structural confirmation of knockout BmNPV bacmids.}

A schematic representation of the verification of gene-knocking out by PCR is shown in panel (a). PCR was carried out targeting the internal sequence of the knocked out gene (INT), upstream (cat-U) and downstream (cat-D) for each KOV. The PCR products were analyzed by agarose gel electrophoresis and the results for five KOVs $(\Delta B m 20, \Delta B m 48$, $\Delta B m 91, \Delta B m 93, \Delta B m 101)$ are presented $(\mathrm{b}, \mathrm{c}, \mathrm{d})$. Lanes 1 to 5 contain PCR products for $\Delta B m 20, \Delta B m 48, \Delta B m 91, \Delta B m 93$, and $\Delta B m 101$, respectively. The results for PCR targeting INT are shown in panel (b). Lanes 6 to 10 contain the PCR products using BmGFP as the template for a positive control of lane 1 to 5. The results for PCR targeting cat-U and cat-D are presented in panel (c) and (d), respectively.

\section{Fig. 2. Four types of KOVs}


The results for BmGFP, $\Delta B m 101, \Delta p k i p(B m 15), \Delta d b p(B m 16)$ and $\Delta l e f-9(B m 50)$ are presented as examples for type-A, $-\mathrm{B},-\mathrm{C}$ and $-\mathrm{D}$, respectively. $\Delta$ ORF indicates the number of open reading frames knocked out in each KOV. Fluorescence micrographs of bacmid-transfected $\mathrm{BmN}$ cells are shown along with a schematic representation.

\section{Table 2. Category (type-A to D) of BmNPV KOVs.}

The knocked out genes (ORF No. or gene name. ORF No. of AcMNPV is parenthesized) and categories are presented. The numbers of ORFs refer to previous reports (Gomi et al., 1999; Katsuma et al., 2011; Ayres et al., 1994). “*” on the ORF No. indicates that there is no repot concerning KOV of the ORF so far. 
(a)

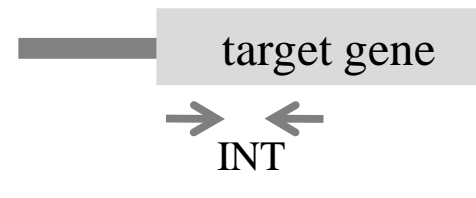

Target gene was replaced with a chlorumphenicol-resistant gene (cat) by lambda red recombination system.

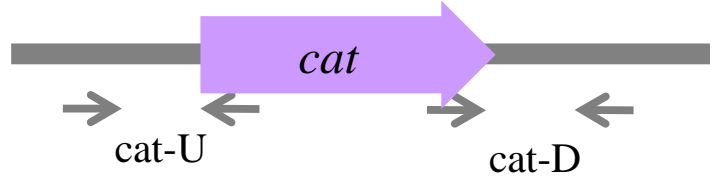

(b)

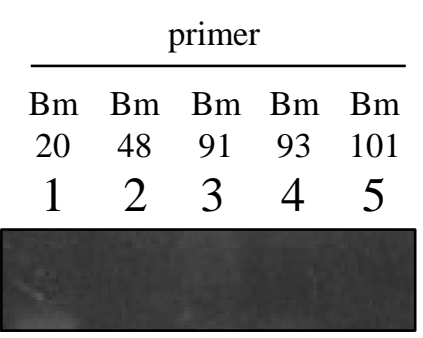

\section{$\begin{array}{lllll}6 & 7 & 8 & 9 & 10\end{array}$}

(bp)

300

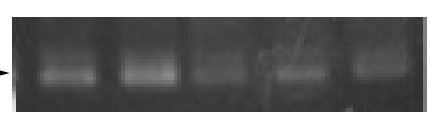

(c)

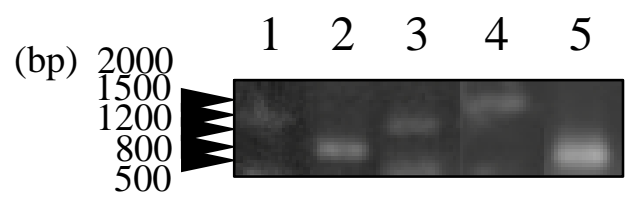

(d)

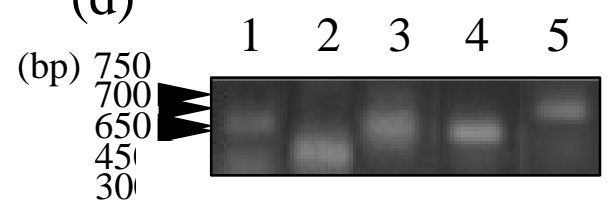

\section{Fig. 1}



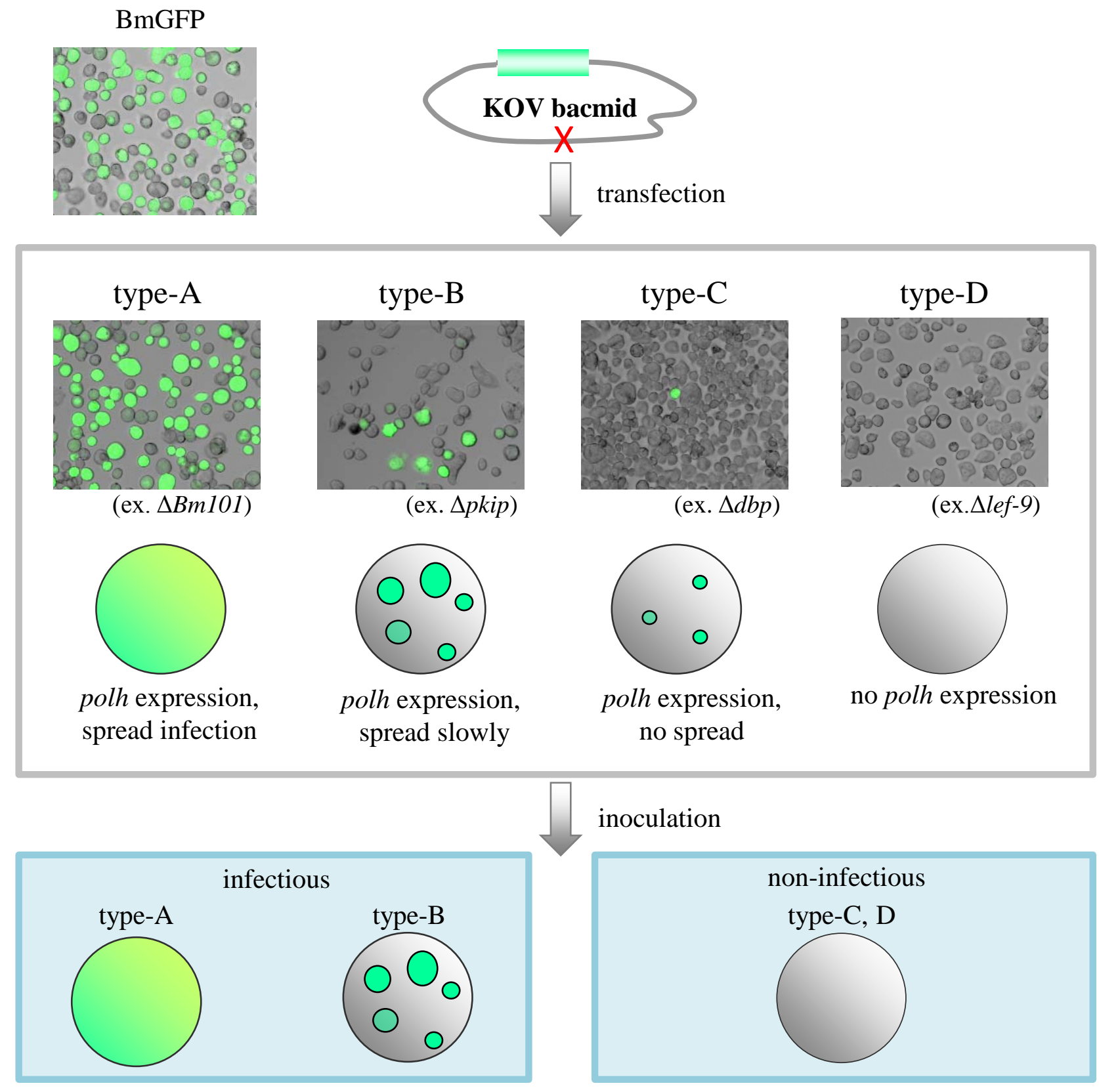

inoculation

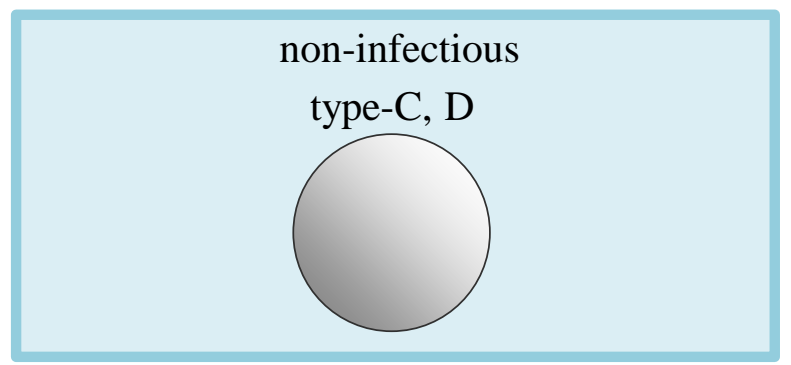

Fig. 2 
Table 1

\begin{tabular}{|c|c|c|c|}
\hline ORF No. & Gene name & 5' primer & 3' primer \\
\hline 1 & polyhedrin & - & - \\
\hline 2 & $\mathrm{p} 78 / 83$ & 5'-GACGAATCGTAAATATGAATCTGTACAATCTTATTTATTCAATAATAGGAgtgtaggctggagetgettc-3' & 5'-AACACTATACATTGTTATTAGTACATTTATTAAGCGTTAGATTCTGTGCGcatatgaatatcetcettag-3' \\
\hline 3 & Pk1 & 5'-ACCACCACCGCCACAAATGCTACGCTGCAAACGCTGGTACAATTTTACGAgtgtaggctggagctgcttc-3' & 5'-AATAATTTTTATGATTTTTATTAACACCACTCACGACAAAAACTCATGTTcatatgaatatcetcettag-3' \\
\hline 4 & & 5'-GCGTGCCTGTACATCAGTATTATTGAGCTTATCATTGCCTTACTGACAACgtgtaggctggagetgettc-3' & 5'-GTGGTGTTAATAAAAATCATAAAAATTATTGTAAATGTTTATTATTTAAAcatatgaatatcctccttag-3' \\
\hline 5 & & 5'-ACGTCCAACAATGGTCGCACATCGTTAAATGGGATTCATTCAAATGCAACgtgtaggetggagetgettc-3' & 5'-TATTTATACGAAATAAATACATACTAATAACTATTATACATGTTTTTATTcatatgaatatcctccttag-3' \\
\hline 6 & lef-1 & 5'-TTTTTTATAACCAAAAATTAACACAATAGAGGTCGAGTTCAAAGGGCAGC gtgtaggetggagetgcttc-3' & 5'-TTTGTCTAGCAATTCCTTTGTTATACAACGAGAAAATTTAGTTCCCTTATcatatgaatatcctccttag-3' \\
\hline 7 & egt & 5'-GAAGCAAAAAATGACTATTCTTTGCTGGCTTGCACTGCTGTCTACGCTTACgtgtaggetggagctgcttc-3' & 5'-CAAACCAAAAAAACCAGGTAAATAATGATGAATAACATTTATTGACGTAAcatatgaatatcctccttag-3' \\
\hline $7 \mathrm{a}$ & & 5'-ATTGTAATGAATCATATAGCACACTTTTAGTTACGTCAATAAATGTTATTgtgtaggetggagetgettc-3' & 5'-TTCGATGTTTTCGCCTTTGAACGTCCTGGCACAATAATGACTGCTCTGGTcatatgaatatcetcettag-3' \\
\hline 8 & bv/odv-e26 & 5'-CAACCAGAGCAGTCATTATTGTGCCAGGACGTTCAAAGGCGAAAACATCGAAgtgtaggetggagctgettc-3' & 5'-ATTAAACGTTTGCCGCTAGCAATCTCGTCCGGATTTATAAACTCGAACCGcatatgaatatcctcettag-3' \\
\hline 9 & & 5'-TTTGAAGGGTGAGGAAGAGCCCAATTGCGTTGAACGCATCACCATAATGCgtgtaggctggagctgcttc-3' & 5'-AATTTAAAAATAAATAAAATAGTTAATAGCTGTCTACCCGTAAAATAATTcatatgaatatcctccttag-3' \\
\hline 10 & & 5'-TATGGAACGTTTGTTAAATCAACTAAATCTTGGCGTTCTACCTTATATTAgtgtaggetggagetgettc-3' & 5'-ATGTATTGTTAGAAAGTTGTGTTGTTTTATTAGTATAACGAAAAAATACAcatatgaatatcctccttag-3' \\
\hline 11 & & 5'-AATGAATAGCGGCGACGACGACGCAACACGACTACACTATTCAAACGCGCgtgtaggctggagctgcttc-3' & 5'-TATTTATTTTTAATAATATTTTTCCAACAAAAAACTTAACACGTTGGTTAcatatgaatatcctccttag-3' \\
\hline 12 & arif-1 & 5'-AGTATTCGATACATATTGCCATTACTTAGTTGTTGAATTAAATATTCAAAgtgtaggctggagctgettc-3' & 5'-ATTACATTAATACTTTATATAATCAAAAATAATACTTTATATAATATTTTcatatgaatatcctcettag-3' \\
\hline 13 & & 5'-GTTTGTATTTCTTTATATAGTATACCAGCCCTTTTATCAGGCATACTTACgtgtaggetggagetgettc-3' & 5'-ACTAACTACTGACGTAACATTTAAACGACTTATTGTTTGTGTTATCATAAcatatgaatatcetcettag-3' \\
\hline 14 & & 5'-ATAAGCCGTACATGTTGGCTTGCTAATTCAGTCAATATCAGACGTTTATCgtgtaggetggagetgettc-3' & 5'-ATTTTAATTATACATGTTTTATTTTATTCTTTCAATAATCATAGGATACAcatatgaatatcctcettag-3' \\
\hline 15 & pkip & 5'-AAAAATGTTGTTTTTTATTATAAGTCAAAATGTCTTGCATATTAACTGCGgtgtaggetggagetgettc-3' & 5'-TTTTATTTATATACTATTTTCTATTAAATATTCCAATGCACACAAATGTTcatatgaatatcctcettag-3' \\
\hline 16 & $\mathrm{dbp}$ & 5'-GAAACACTTCAATTAGACTTGAACCACAGCAGACAGCGCACGTCGGTAGCgtgtaggctggagctgcttc-3' & 5'-ATGCAAGACATTTTGACTTATAATAAAAAACAACATTTTTATATATTCATcatatgaatatcetcettag-3' \\
\hline 17 & & 5'-GGTTCAAGTCTAATTGAAGTGTTTCACAGAATATAAGATATATAATAAATgtgtaggctggagctgcttc-3' & 5'-TTTAACTCGTTAAAGTTACGGTTAAAATGAGCACGTTTGGGTCGTTTTGGcatatgaatatcetcettag-3' \\
\hline 18 & iap1 & 5'-AATGAACGAGGACACTCCTCCGTTTTATTTTATCAACACGCGCGACAACTgtgtaggctggagetgettc-3' & 5'-TTATAAAATCGGTGACGTCTTGACGACAGGTCGGACACTTTTGATCTAATcatatgaatatcctcettag-3' \\
\hline 19 & lef-6 & 5'-TAAAATGGTGTTCGACGTGTACTACAACGGCTATTATGTGGAAAAAAAATgtgtaggctggagctgettc-3' & 5'-AATACATGTTTTATTGTTTTTCTAATACATTCAAGTCGTTTAGATGATTAcatatgaatatcctccttag-3' \\
\hline 20 & & 5'-CTGTTTTACTTCATCTGTATATTTTTTCAAGATGTTCTCAAAGAATTACAgtgtaggctggagctgettc-3' & 5'-TTAAAAATTATTTATTCCGTAATTAAGAAAATTTTGTCTTGACTTCATAAcatatgaatatcctccttag-3' \\
\hline 21 & & 5'-TAAAATACTTTATTCCATTTGATTTTTTTTATTTATGTGTATAACATATTgtgtaggctggagctgcttc-3' & 5'-CATCTTTTTATCCAAATTAGGAGTTAATTATTATTCATTTGTATCGCGACcatatgaatatcctcettag-3' \\
\hline 22 & bro-a & 5'-AATAAATTTAATTTAATAAATTAAAATGGCTCAAGTTAAAATgtgtaggctggagetgettc-3' & 5'-TTAAAATTGTTATTCAATCGTGCAATCTGACGTTCCGTCATGCTCGACTGcatatgaatatectcettag-3' \\
\hline
\end{tabular}


Table 1 Continued.

\begin{tabular}{|c|c|c|c|}
\hline 23 & sod & 5'-AGTAGGGTGACTGTTTGCTCAAAATTAAAATGGTGTAATTAAGAAACAACgtgtaggctggagctgcttc-3' & 5'-AAAGCGATGACATCATTTACATATGGCAATTATTCCGCATCCCAAACGGCcatatgaatatcctccttag-3' \\
\hline 24 & fgf & 5'-CAAATTTTTATATAAAGGGGCCACTTTGCTATGGGGAATTAAAATTCGTCgtgtaggctggagetgettc-3' & 5'-ATAAAATAATTATTTAAATAAAAATGTTTTTATTGTAAAATACACATTGAcatatgaatatcctcettag-3' \\
\hline 25 & & 5'-TTTTACACTATTACTTATAAATGACAACGGTTGCTGTGAATGTGCCCTTGgtgtaggctggagctgettc-3' & 5'-CTAGTTAATAATTTGTGTGATCAGATAACTATTAACGTCCACATGGTATTcatatgaatatcctccttag-3' \\
\hline 26 & ubiquitin & 5'-TTATAAGTAATAGTGTAAAAATGCAAATATTCATCAAAACATTGACGGGCgtgtaggctggagctgcttc-3' & 5'-ATAAAAAACTTTGTACATTTAATGATTTTTATTATTATTATTATTATTATcatatgaatatcctccttag-3' \\
\hline 27 & $39 \mathrm{k}$ & 5'-ACATGCCGGAGCAACAATCTTCTACAGAAACTGCGGCCGTGTGCAAAAATgtgtaggctggagctgcttc-3' & 5'-ATAATAATAATAATAATAATAAAAATCATTAAATGTACAAAGTTTTTTATcatatgaatatcctccttag-3' \\
\hline 28 & lef-11 & 5'-GCACGCACTTAGGCGGGTGTAATTCGGACTGCTTGACCCGAAGCGAAATAgtgtaggctggagctgcttc-3' & 5'-TTTGTGATGAAGACACACCTTACGCTTAGAACATTTGTCGGTAATTACTGcatatgaatatcctccttag-3' \\
\hline 29 & & 5'-TTAAACGATGGTGCTCGTGTGCGCAATTTTTTAAGTGTCAAATATGTAAAgtgtaggctggagctgcttc-3' & 5'-AAATATTTGTTGAGCGGCACGATGGCCAGGCTGCGCGTTATTTCGCAATTcatatgaatatcctcettag-3' \\
\hline 30 & $\mathrm{p} 43$ & 5'-GCCAATCATGGACAAACGTGCCAATTCTCGCAAACCCTTTCTGTTCTACAgtgtaggctggagctgcttc-3' & 5'-TAAAAAATTGCGCACACGAGCACCATCGTTTAATACCTGATTGCAACTATcatatgaatatcetcettag-3' \\
\hline 31 & $\mathrm{p} 47$ & 5'-CGCGTTATTTTCCATAATTATGTTTGTCACCCGGTTGGAGCACACCACAC gtgtaggctggagctgcttc-3' & 5'-GATTGGCTCAGTATAACGACTGGTCAAAATTGACAACGCGTTTGCCGTAAcatatgaatatcetccttag-3' \\
\hline 32 & lef-12 & 5'-ACACGTAGAATTCAACAGACGACTAGAGTACGTAGGCACAATCGCCACAA gtgtaggetggagctgettc-3' & 5'-ATTAAACTACGGTTATTGCGCTAAAATATCACATTTAACACGCACATTATcatatgaatatcctcettag-3' \\
\hline 33 & gta & 5'-TTTAGCGCAATAACCGTAGTTTAATCGAAGAGAATAGCCGTCTCGCCACAgtgtaggctggagctgcttc-3' & 5'-GGCGACTTACCAGCTACAAGTTGAAAAATTTTTTAATGTCTTCGTAATTGcatatgaatatcetcettag-3' \\
\hline 34 & & 5'-CTGGTAAGTCGCCATGAACACCCGATACGCTACTTGCTATGTTTGCGACGgtgtaggetggagctgcttc-3' & 5'-GTTGTTGAGCGCGGCGGTTGGGGTAAATTGGAATCGAAATTCCGTTGGTAcatatgaatatcctccttag-3' \\
\hline 35 & & 5'-GACGCAAGCGCTTCGAGTTTTGGCTCGCTCGCTACCTCCGCTGTACGACTgtgtaggctggagctgcttc-3' & 5'-GTTACAAAGTTTTGTATTTCAATTTATATCTAATGCCGCTTTTACATTTAcatatgaatatcctcettag-3' \\
\hline 36 & & 5'-CATGTTGCCCTACGAAATGGTGATTTCCGTGTTGGTTTACTTGTCGCCGGgtgtaggctggagctgcttc-3' & 5'-GTCGTATGTTGCTTATTATTGATACAAAGTTTTCTTAATTGTAAACACACcatatgaatatcctccttag-3' \\
\hline 37 & odv-e66 & 5'-TTATTGTCGTAGTTGTAATATTTTTAATATGTTTTTGGTGCCTATTAAATgtgtaggctggagctgettc-3' & 5'-CTTTTGTGTTAACAATCAGTCTTTTGAATATTACATTCTCCCTAAATGTAcatatgaatatcctcettag-3' \\
\hline 38 & ets & 5'-ATATATATGTATGCAACAACTTTTTAATATTCACATTAAAAAGTGCCAGGgtgtaggctggagctgcttc-3' & 5'-AGACTGATATTGTTGTTGTTGTTGTTACAAAATAGAAAATAAAAAATATAcatatgaatatcetccttag-3' \\
\hline 39 & lef-8 & 5'-AATTAGTGTGCAATAATCGCTTTACGATGACGGACGTAGTTCAAGATTTCgtgtaggctggagctgcttc-3' & 5'-TTTTCGTGCACAGATATAATTTTATCATGTTTGCAATCGTGCAAGAGTTAcatatgaatatcctccttag-3' \\
\hline 40 & & 5'-CGTAAAGCGATTATTGCACACTAATTATGTCTAAACGTGTTCGTGAAATGgtgtaggctggagctgcttc-3' & 5'-GATTTTAAAGAGTTTTTTAAATCCTCAATGTTCTTGCTTATTATGTCAATcatatgaatatcctcettag-3' \\
\hline 41 & & 5'-AATGACGTGGCCTTATTCCAATAGTTTTAAACTTATCAAAGCTTATCAAAgtgtaggctggagctgcttc-3' & 5'-ATTAAAATGTCGAACAAAGGAAAAAAACAATTGTAACAAAAATAATTTACcatatgaatatcctccttag-3' \\
\hline 42 & $\mathrm{Bm} 42$ & 5'-TATGTTCTGCACCGTTTGTTTAAAAGACAGAAATTACTATATGTTCAAACgtgtaggctggagctgcttc-3' & 5'-CCTTTGGTTTCTCGAAGCATCGCGTTGCATTCCTCTAGCGTTTTTGGGGGcatatgaatatcetcettag-3' \\
\hline $42 \mathrm{a}$ & lef-10 & 5'-CCCTGGACATTGAACTCGATTTTAGGAATTTTTTTAAAATGCAATCATGAgtgtaggctggagctgcttc-3' & 5'-TTCACCGAGGCACAGAGGTCTAACTTGATCGGTTTCTTGGTCGAACACATcatatgaatatcctccttag-3' \\
\hline 43 & vp1054 & 5'-CAATTATTCAGATCCCGATAACGAAAACGACATGTTGCACATGACCGTGTgtgtaggctggagctgettc-3' & 5'-AATAAGAATGCTTGTTTAACAAATAGGTCAGCTGTTAAATATTGGAGATGcatatgaatatcctccttag-3' \\
\hline 44 & & 5'-TTAAACAAGCATTCTTATTTCAATAATTGGAGACAGTTGTATCCACAATTgtgtaggctggagctgcttc-3' & 5'-GTCAATATTTTTTCACCAATTTGTCAAATTTATTGTAATCTCGCTTGCCGcatatgaatatectcettag-3' \\
\hline 45 & & 5'-CATGTTGCGATCAATCATACGACGTTTCAAGAGTACAAACAATCTCAGCAgtgtaggctggagctgettc-3' & 5'-ATATCTAGCGCAATTAAATGAAACCATAAAAATACAAACAACAAACTTTAcatatgaatatcctccttag-3' \\
\hline 46 & & 5'-CGGTTTATTTCACTCGAAGGTATTATCTCAGACAGTCGAACGTGCGCGCAgtgtaggctggagctgcttc-3' & 5'-TAAATTAATTTTTTTTTTACTTGTACAGTTTTATTTCTAGTTCACGATCCcatatgaatatcctcettag-3' \\
\hline
\end{tabular}


Table 1 Continued.

\begin{tabular}{|c|c|c|c|}
\hline 47 & & 5'-TTCTAATTACAATGTATCAAATTCCCGATATGTTATACAATGAAAAAATGgtgtaggetggagctgcttc-3' & 5'-AAAAAAAATTAATTTATTAATAGTTGTAATAATTATCTTCGTCCTCATCTcatatgaatatcetcettag-3' \\
\hline 48 & & 5'-AATAAAACACACACACACACAAAGTTTTTATTATATTGTCTTTTATTGATgtgtaggctggagetgcttc-3' & 5'-TGTAATTAGAATTAAGCAATTTCGTCTTCGGTTTCTGTATCTGTAGTGGAcatatgaatatectecttag-3' \\
\hline 49 & $\mathrm{fp}$ & 5'-TTACGCACCATATACGCATCGGGTTGATATAATTAATATGGATCAATTTGgtgtaggctggagctgcttc-3' & 5'-TTCTTACATTACGAGATTCAACTTGATACTAAAATTAATTATCACTTAAAcatatgaatatcctccttag-3' \\
\hline 50 & lef-9 & 5'-CTCTTATACTTTGCACTTTGCGTTAATACGTGTTCGTGTACAGACGTAATgtgtaggetggagetgettc-3' & 5'-AATTAATAAAACACTGATTGCATCTAATTAAATACGCTTTATTTATATTAcatatgaatatcctccttag-3' \\
\hline 51 & & 5'-AAAGCGTATTTAATTAGATGCAATCAGTGTTTTATTAATTTTAAAGCAACgtgtaggctggagctgettc-3' & 5'-GGGCATTTTTTTTAATCCAGGATTTTAATAAAATAAAAACAAAATTTTAAcatatgaatatcctcettag-3' \\
\hline 52 & gp37 & 5'-GTTACGACAGCGGTTATTATTCCGATTAGTGTTCTATAAGTATAATCATAgtgtaggctggagctgcttc-3' & 5'-AATCCTGGATTAAAAAAAATGCCCCAATCCCAAGTTTTTGACACCTATGAcatatgaatatcctccttag-3' \\
\hline 53 & dnapol & 5'-ATTTAAAAATGAAAATATATTCTTACAATGAACTCAAAACGCGCTTTGCAgtgtaggctggagctgcttc-3' & 5'-ATAACACCTTACAGTAACATACAATAAAACAACATAGTATCGTATATAATcatatgaatatcctcettag-3' \\
\hline 54 & & 5'-CATAAATACCATGAGTGCTCGAATCAAAACTCTGGAGCGGTATGAGCACGgtgtaggctggagctgcttc-3' & 5'-TTCTATTCGACGTTTGGTTGAACGCTGGCCGCTGTTGTTCGCGCCAACGTcatatgaatatcctccttag-3' \\
\hline 55 & lef-3 & 5'-ATTGACAACAACAGCAATATGGCGACCAAAAGATTTTTTTCTGGAGAAAG gtgtaggctggagctgcttc-3' & 5'-AATTACAAAAATGTATAATCATTTTCATCTTCGTCATACTTCAACAATCCcatatgaatatcctccttag-3' \\
\hline 56 & & 5'-ATTGCTGTTGTTGTCAATATGTGGGAATCTATACGATGGCAAATACTGAAgtgtaggctggagetgcttc-3' & 5'-GGTCGTTTGATCGTTAAATCGAGCGGGTCTGTTGAGAGCAGCTCTTATTCeatatgaatatectcettag-3' \\
\hline 57 & & 5'-ACTCAAAGATAATTTAAACACGTTCAGCAGCAAGTCGGTGGTTTGCGCTCgtgtaggctggagctgcttc-3' & 5'-CATGTTGAATGATGCCTGATTTGAAGCCGCTGGCTTTTTATATACACACGcatatgaatatectcettag-3' \\
\hline 58 & iap2 & 5'-TCATTTACGATGTCGACAATTATTCTTTCAAAAATGATAGTGTGCAATAAgtgtaggetggagctgettc-3' & 5'-ATAATTATTAATAGCTTATTTTTAAAGAATGTCGTAGTCATTTGCAATGTcatatgaatatcctcettag-3' \\
\hline $58 \mathrm{a}$ & & 5'-AATGACTACGACATTCTTTAAAAATAAGCTATTAATAATTATTGCATTGTgtgtaggctggagctgcttc-3' & 5'-CATTATGTACAATAATATGGGTTTTTATTTTACAACATTTATGTATATGAcatatgaatatcctccttag-3' \\
\hline 59 & & 5'-ACCAATATCTAAACAGCGACACGCCCGATTACACCATAGAATCCTTAAACgtgtaggctggagctgettc-3' & 5'-ATAACAATTGTAATTTCATATACATAAATGTTGTAAAATAAAAACCCATAcatatgaatatcctccttag-3' \\
\hline 60 & & 5'-GATAAATAAAAATGAAAATAAATTTGTGCAATATACAATTTCAGTCTTTAgtgtaggctggagctgcttc-3' & 5'-ATCCAATTTCGTGTTCAAGAAATTGGTGATGATATCTTTGAACGTGCACGcatatgaatatcctccttag-3' \\
\hline 61 & & 5'-TAATAAGTGCTCGTTAAAATGTCCAATTTAATGAAAAACTTTTTCACCGAgtgtaggctggagetgettc-3' & 5'-TTTTATTTATCTTACTATTAATACGCCGGCAATTGGTATGCTTCGTCCAGcatatgaatatcctcettag-3' \\
\hline 62 & & 5'-AGTAAGGGAATAAAAATGAATTTATATTTGTTGTTGGGCGCACTGACCATgtgtaggctggagctgcttc-3' & 5'-TTTAACGAGCACTTATTATCAATCTATTGAGCTGGTATTTTTGTTTAAAAcatatgaatatcctcettag-3' \\
\hline 63 & vlf-1 & 5'-ACAATGAACGGTTTTAATGTTCGCAACGAAAACAATTTTAATTCTTGGAAgtgtaggctggagctgcttc-3' & 5'-TTTTATTCCCTTACTCTATTCGTTGCGATAGTACAACAACGATTCTCCCGcatatgaatatcctccttag-3' \\
\hline 64 & & 5'-ACATGAATTTGGACGTGCCATACTATCGGTTGGGCAACCACGAAAAGGTGgtgtaggetggagctgettc-3' & 5'-AGGAGAATCAGTGTCAATACTATCCGAATATTGTTGTTGTTCTCTTAATAcatatgaatatcetcettag-3' \\
\hline 65 & & 5'-CGCACTGTATAATCATGGCGACGACTCTGTACACCAACAAGGTGTGGTGCgtgtaggetggagctgettc-3' & 5'-GTTTACAACTTATTTGCTAACAGAAATTTATGCAACAAAAGTGGTTTGGCcatatgaatatcctcettag-3' \\
\hline 66 & gp41 & 5'-ACGTGGCAATTTTTATTACAACACCCCTCCGCCGCCGCTGAGGTATCCCTgtgtaggctggagctgcttc-3' & 5'-GATTATACAGTGCGTCCTTTCGTGTTTGGCCGAGTGACGTTAGGCGCGGCcatatgaatatcctccttag-3' \\
\hline 67 & & 5'-ACAACGCTGAACAAAATTAAATACGATAGTGAACTGTTGCTTCATTATTTgtgtaggctggagctgcttc-3' & 5'-ACTTTTTATTTGTACTCTTATGATTACAAAACTCAATACACGGATTACTTcatatgaatatcctccttag-3' \\
\hline 68 & & 5'-GGGCGTTGGCGCCCATATTATCAAGGTGGCTAGCTCGCAGCAACTTAGATgtgtaggetggagctgettc-3' & 5'-CTACGGCGCGTTTGGGCGACGACGTGTTTACAGCAGCGTCCGTCTTATTAcatatgaatatcctcettag-3' \\
\hline 69 & p95 & 5'-TTTTCTTATAATAGCGTTTACTTTAATTTATTTGGCAATTTATTTTAAATgtgtaggetggagctgcttc-3' & 5'-ATTTGTTTTCGACATAAAATGTTTATACAATGGAATCTTCTTGTAAATTAcatatgaatatcctccttag-3' \\
\hline 70 & vp15 & 5'-TATATAAACGCTATATAACAGTTTTTGCTAGTGTTATTTTATACAACATTgtgtaggctggagctgcttc-3' & 5'-TTAATTAATTGTTTACATAACATTCCACTTTAATGTAATAATATTCTTTAcatatgaatatcctccttag-3' \\
\hline
\end{tabular}


Table 1 Continued.

\begin{tabular}{|c|c|c|c|}
\hline 71 & $\operatorname{cg} 30$ & 5'-AAATGGAGTTTGTCAAATTGCAATGCAACATTTGTTTTTCGGTTGCAGAAgtgtaggctggagctgcttc-3' & 5'-TTAATTAATCTACATTTATTGTAACATTTGTGGTGATAGTAGTAGCGTTTcatatgaatatcetcettag-3' \\
\hline 72 & vp39 & 5'-ATTTATAACGGCAACAATATGGCGCTAATGCCCGTGGGTATGGCGCCGCGgtgtaggctggagctgettc-3' & 5'-TTTTAGGCGGCTACACCTCCGCCTGCTTCGCCGAGAACAACACCAGGCGCcatatgaatatcctccttag-3' \\
\hline 73 & lef-4 & 5'-ATTGTTGCCGTTATAAATATGGACCACGGCAATTTTATGATTGAGAAAGAgtgtaggctggagctgcttc-3' & 5'-TTTGGCACGATTCGGTCGCGACGATGTTTCAACACGTTTATTGTCGTGTCcatatgaatatcctcettag-3' \\
\hline 74 & & 5'-TACAAGTTTTTGTTTGATTCTTTTTATTAAGCAACATGTGGTACATTGTTgtgtaggctggagetgettc-3' & 5'-AGCACGTTAAGCGGATAGAACGGGCAGTCTGAGCTGTTAAAGTCAATACAcatatgaatatcctccttag-3' \\
\hline 75 & $\mathrm{p} 33$ & 5'-TCTTCGCAAGCAACTTACTTATACCATTTTGCGTGTTTGATGAAATACAAgtgtaggctggagctgcttc-3' & 5'-GTTGCTTAATAAAAAGAATCAAACAAAAACTTGTATTATTGCAAATTTAAcatatgaatatcctccttag-3' \\
\hline 76 & & 5'-CGCCGACGAGCCTATTATTTATTTCGAAAATATTACAGAATGTCTTACGGgtgtaggctggagctgcttc-3' & 5'-AATGTTTCAAAAGTACACTTAAATTATCGTTTTCCCAATTGACGGGCACGcatatgaatatcctcettag-3' \\
\hline 77 & odv-e25 & 5'-AACAAATCATGTGGAAAATCGTGTTATTGATCGTTTTGCTCGTACTGATTgtgtaggetggagetgettc-3' & 5'-TAAATAATATATAAATAGACTTTTTTTGTTTTATTAATTCATTTCTCTGTcatatgaatatcctcettag-3' \\
\hline 78 & dnahel & 5'-TTTACAATTTTTTTTAAAAGACGTGCCTGAAGACAAAACGTACGAGATTAgtgtaggctggagctgcttc-3' & 5'-ACAGAGAAATGAATTAATAAAACAAAAAAAGTCTATTTATATATTATTTAcatatgaatatcctcettag-3' \\
\hline 79 & & 5'-TTTGGCTATCGTGTTTATATTTTTCGTGTTAATTTATTTAATAATTTCGAgtgtaggetggagetgettc-3' & 5'-TTAATAAACTTGGTCTGTAGATGAAACATTTTGGTTGTCCAAGTCCACGTcatatgaatatcctcettag-3' \\
\hline 80 & bro-b & same as bro-a 5 ' primer & 5'-TTACAACGACGCGTGGCGATTCGACGAAAGCGCGAAACAAACTGAACGGAcatatgaatatcctcettag-3' \\
\hline 81 & bro-c & same as bro-a 5 ' primer & 5'-CACTTTGCTGGTCACCCAATGCTTAAACGCTTGACGACATATAAAAATTTcatatgaatatcctcettag-3' \\
\hline 82 & $38 \mathrm{k}$ & 5'-GTTCTCGTTTTAAGCGAGTACGCAGACCTCAAATACCTTGGCTTTGAAAAgtgtaggetggagetgettc-3' & 5'-AATGCATAATAAATAAAACATTTTGTAATTTAATACTGTTTTTTATTAACcatatgaatatcctcettag-3' \\
\hline 83 & lef-5 & 5'-TTTTCAAAGCCAAGGTATTTGAGGTCTGCGTACTCGCTTAAAACGAGAACgtgtaggctggagctgcttc-3' & 5'-CAGCCAGACATTCCACACATCCGACAGTAGCGAAGGAACGAAGCGATTTCcatatgaatatcctccttag-3' \\
\hline 84 & p6.9 & 5'-TAAGGTAAAAACTACAGCTACATAAATTACACAATTTAAACATGGTTTATgtgtaggctggagctgcttc-3' & 5'-TAGTAGCGTGTTCTGTAACTTCGGCGGCTTGTCAATGAACGGCTCCTGGAcatatgaatatcctccttag-3' \\
\hline 85 & $\mathrm{P} 40$ & 5'-GTGTGCGTCGTCGGTCACGATGAGCGCTATCGCGTTGTATTTGGAAATAAgtgtaggetggagctgettc-3' & 5'-GTTTAAATTGTGTAATTTATGTAGCTGTAGTTTTTACCTTATTAATATTTcatatgaatatcctcettag-3' \\
\hline 86 & $\mathrm{P} 12$ & 5'-TATCGATGATATGGACACTGGCAACAACATGTCGCAAACACGAAGAAGCCgtgtaggctggagctgettc-3' & 5'-CGTGACCGACGACGCACACTTACTCTGTAACTATCACTTGGATCGTGTTGcatatgaatatcctcettag-3' \\
\hline 87 & P45 & 5'-TTATAAGGTTATATTGAATGCACCATGTGCGCTTACAGATTACAATACAGgtgtaggctggagctgcttc-3' & 5'-TGATGTGCCTTATAAATGATTCGGGCACAATCATTTCTGTCATTAGCACGcatatgaatatcctcettag-3' \\
\hline 88 & vp80 & 5'-GGTGCATTCAATATAACCTTATAATATGAACGATTCCAATTCACTGTTGAgtgtaggctggagctgcttc-3' & 5'-TTTTTTATTATATAACATTGTAGTTTGCGTTCATTAACATTATTAGTCTTcatatgaatatcctcettag-3' \\
\hline 89 & he65 & 5'-TTTGAGGCATATAAGGCTTGATACAGGCACAGTAAGCAACGCTGCTAACgtgtaggctggagctgcttc-3' & 5'-ATTTTTAATTTTTATTTATATGCGACACAAATGGATAAATTTGATGGTACcatatgaatatcctcettag-3' \\
\hline 90 & & 5'-CGTCAATGAAACAACGTGTAGTATTTTTTACAATATTTAAGTGAAACATTgtgtaggctggagctgcttc-3' & 5'-TTACAAAGAAAACAAAGGCAATAAATGATTCTTTTTTTCAAGTTTACGCAcatatgaatatcctcettag-3' \\
\hline 91 & & 5'-TTAAGAAGCACATTATGAAACCGACGGCGGCCGACATTATATCAAGAGTGgtgtaggctggagctgettc-3' & 5'-TTATGTTGTTGCATTTCTATTTCTAATATCATAATTTTCTAATAAAGTAGcatatgaatatcctccttag-3' \\
\hline 92 & odv-ec43 & 5'-GTTCACAAATTAAAATCTAAAGTCTAATGAAGTAAGACATATACATCGCGgtgtaggctggagctgcttc-3' & 5'-AATGTGCTTCTTAACTACAAATAATAGTTGTACTTGATGGGCGTCACCGTcatatgaatatcctccttag-3' \\
\hline $92 \mathrm{a}$ & & 5'-AAACGTATAAAAGTATTATGTGTTTTATTTAACTAGCATAAGATTTAAAGgtgtaggctggagctgcttc-3' & 5'-CGCGATGTATATGTCTTACTTCATTAGACTTTAGATTTTAATTTGTGAACcatatgaatatcctccttag-3' \\
\hline 93 & & 5'-ACAATAAAAAATTAAAACATATGCATCAGTGATATTGCCTGTGCTGCACTgtgtaggctggagctgcttc-3' & 5'-ATCTTTAAATCTTATGCTAGTTAAATAAAACACATAATACTTTTATACGTcatatgaatatcctccttag-3' \\
\hline 94 & & 5'-CCAATTTGTATAGACGCGTTTACAACTGAAATGTAAGAGATTCTGTCAACgtgtaggctggagctgcttc-3' & 5'-GTAATTGATTAATATGTCGTACAGTTTGGCGGCGCTGTGTTTGCACACAAcatatgaatatcctccttag-3' \\
\hline
\end{tabular}


Table 1 Continued.

\begin{tabular}{|c|c|c|c|}
\hline 95 & & 5'-TCTTCACACTCAATTAAGACGATCATGTTGAACTTTTGGCAAATACTTATgtgtaggctggagetgcttc-3' & 5'-GTTGACAGAATCTCTTACATTTCAGTTGTAAACGCGTCTATACAAATTGGcatatgaatatcetcettag-3' \\
\hline $95 \mathrm{a}-96$ & & 5'-TAAACCTGGGTCTATATAACTCGGCGTCGGCCGAGTTTATTTTTAACATTgtgtaggetggagctgcttc-3' & 5'-TCTTTAATTATTAAATAAAACAAAATGATATAATAAAATTTGGTATTTATcatatgaatatcctccttag-3' \\
\hline 96 & & 5'-TTATAATACATATTTGAAAAATTACAGTGTAATTGAAGGTGCGATGTGTTgtgtaggctggagetgettc-3' & 5'-TCTTTAATTATTAAATAAAACAAAATGATATAATAAAATTTGGTATTTATcatatgaatatcctccttag-3' \\
\hline 97 & & 5'-CAAAATATCTCGTACGTACAAAAATACTCGGACATTCTCCAATAAGTAAAgtgtaggctggagctgcttc-3' & 5'-GTCTATTTGTTGCCCTTGGGTCATGGCGCCTGTAGTAATCGTTCTTATTGcatatgaatatcctccttag-3' \\
\hline 98 & & 5'-TAACAACATGAGCATTTTAAACGTTGTAGAAGCGTGCGATTTGGCACACAgtgtaggctggagctgcttc-3' & 5'-TATTTTTGCACACAAAATATTGAACTCGTTATGATTTCATCATTTGATAGcatatgaatatcctccttag-3' \\
\hline $98 \mathrm{a}$ & & 5'-CTATCAAATGATGAAATCATAACGAGTTCAATATTTTGTGTGCAAAAATAgtgtaggctggagctgcttc-3' & 5'-ACACGAAGACAAATTGATTCAATGTAAAATTTATTTGTAATAAATTATATcatatgaatatcctccttag-3' \\
\hline 99 & & 5'-AAGCGACCCATATATTGTCGAATATAGAACACCATGAAGCTGATTATCCTgtgtaggctggagctgcttc-3' & 5'-TCAGCCCCTCGGGATGGCCAAAATGTGCCGTAGTTTTTAATGGATCTCTCcatatgaatatcetcettag-3' \\
\hline 100 & Pk2 & 5'-TGGGAGCTCGTGTTTGAATTTAGTGACGCGTTAACACTTAATTGTATACCgtgtaggctggagctgcttc-3' & 5'-GGTGTTCTATATTCGACAATATATGGGTCGCTTTTAAATCGCCTTGTCCCcatatgaatatectcettag-3' \\
\hline 101 & & 5'-AAGCATTTGTATATAATCAATTGAATTGTGCACTAATATAAGAATTAAAAgtgtaggctggagctgcttc-3' & 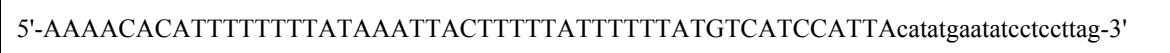 \\
\hline 102 & lef-7 & 5'-AAGCAAGTATCATTTGTGTGCAGTTGTTATTAGGATGTCGAGCGTTACAAgtgtaggctggagctgcttc-3' & 5'-TAATGGATGACATAAAAAATAAAAAGTAATTTATAAAAAAAATGTGTTTTcatatgaatatcctccttag-3' \\
\hline 103 & chitinase & 5'-TAAACGTTTTGTGGTTGGTGGTCGCCGTTTCCAACGCGATTCCCGGCACGgtgtaggctggagctgettc-3' & 5'-CTCTAAGTTTAAACTGTGCGTTCATCGCGTTGAGCAAGTCGCCGTTATCGcatatgaatatcctccttag-3' \\
\hline 104 & v-cath & 5'-TTTAATTTGTCTTAATTTTAAGATGTAATTATTTTATGTAAAAAAAAAATgtgtaggctggagetgettc-3' & 5'-TAATTACAAAATATCTTAGGCGATATGATTATGTTCCAAATAGCGAGAGAcatatgaatatcctccttag-3' \\
\hline 105 & gp64/67 & 5'-GTGCCTTGTGTCACGTGGCCAAATAGCGGTCGGGTATATAAGATGCCTCAgtgtaggctggagctgettc-3' & 5'-TTTAATATTGTCTACTATTACGGTTTCTAACCATACAGTACAAAAATAAAcatatgaatatcctcettag-3' \\
\hline 106 & p24 & 5'-GCATCTTATATACCCGACCGCTATTTGGCCACGTGACACAAGGCACGTTGgtgtaggetggagctgcttc-3' & 5'-TGTCGAAATCGTACAAAACCCTTATTATTATTTATTCAGGCACATTAAATcatatgaatatcctccttag-3' \\
\hline 107 & gp16 & 5'-TAATAAGGGTTTTGTACGATTTCGACAATGAACTTTTGGGCCACGTTTAGgtgtaggetggagctgcttc-3' & 5'-ATTGAACGTTAAGCACGTTGTTAACTACCCCGTTTTTTGTTAATAGCACGcatatgaatatcetcettag-3' \\
\hline 108 & pp34 & 5'-CACTAAGTTAATATACTAAAAATCATATAGTCGCTACAATATTTTGAAATgtgtaggctggagctgcttc-3' & 5'-GCTTATTTTGTTGTCAACGTTCCCGTGAGTATATTTTTGATATCGTTAACcatatgaatatcctccttag-3' \\
\hline 109 & & 5'-GCATGTCCGACAAAACGCCGACAAAAAAAAGCGGCGGCCATGCCATGACGgtgtaggctggagctgcttc-3' & 5'-GATGTCGACGTGCTAACTTTACCAAATCTAAAGACCTAATGGTAAAATGGcatatgaatatcctccttag-3' \\
\hline 110 & alk-exo & 5'-TTTAACAATTATGTGAAAACGATCGAGTTGAGTCGAGCGCAGTTGTCTCGgtgtaggctggagctgcttc-3' & 5'-GAATATAATTAAAGATTTATCATTTTATTTCCAAATAATATACACAATTGcatatgaatatcctccttag-3' \\
\hline $110 \mathrm{a}$ & & 5'-ACGCGGACGCTTTGCAACATTTGAAATTTTTGAAAGTAAATTATAACAAAgtgtaggctggagctgcttc-3' & 5'-TAAAATGATAAATCTTTAATTATATTCTTTTATTTATACATTAATTTTATcatatgaatatcctcettag-3' \\
\hline 111 & & 5'-CAATCCACTCCACAGCGTCCAAAACGGACATATTTTTATATACACGAGCGgtgtaggetggagctgettc-3' & 5'-TTTATAATATAAACGTTCACGTTACGACGCGCGTGTAATTTTCGGGACCAcatatgaatatcctcettag-3' \\
\hline 112 & $\mathrm{p} 35$ & 5'-ATTGCAAAATGTGTGTAATTTTTCCGGTAGAAATCGACGTGTCCCAGACGgtgtaggetggagctgcttc-3' & 5'-ACACAATCAAGCAATGACAAAGAATAATATTAGGCAATAAATTTTAACATcatatgaatatcctccttag-3' \\
\hline 113 & p26 & 5'-GATAATTAAATAATTTATTTGCAAGCTATACGTTGAATCGAACGGACGTTgtgtaggctggagctgcttc-3' & 5'-TATAAGATTTTAATTAATATACAAATAATTTAATAATAATTCTTATTTAGcatatgaatatcctccttag-3' \\
\hline 114 & $\mathrm{p} 10$ & 5'-TTGTATATTAATTAAAATCTTATACTGTAAATTACATTTTATTTACTATCgtgtaggctggagctgcttc-3' & 5'-ACGACGAGCGTCTGAATCGAATTCAAAAGCTTGGATTTTCAGCGTAGGCT catatgaatatcctcettag-3' \\
\hline 115 & $\mathrm{p} 74$ & 5'-TCTAATAAATATTTCATTATTATCGTTGTTTAATTTAAGTCATATATAACgtgtaggctggagetgcttc-3' & 5'-CAGACGCTCGTCGTGGTAAACGCAGTTCCAAGTAAATAAATCGTTTTTTTcatatgaatatcctccttag-3' \\
\hline 116 & me53 & 5'-TGAACGTGCACAGTATTGTGTTGATTTTTGAGTGCTAACCAACAGTTACAgtgtaggctggagctgcttc-3' & 5'-CAATAACGATACATTTTATTTAGACATTGTTGTTTACAATATTAATTAACcatatgaatatcctcettag-3' \\
\hline
\end{tabular}


Table 1 Continued.

\begin{tabular}{|c|c|c|c|}
\hline 117 & ie- 0 & 5'-GCTTGCTTGCGCGCTGGATAGTATAAGTGATTGATAACGAGCAACGCAACgtgtaggctggagctgettc-3' & 5'-TGGACACTTTGCGTGCGTGGTGGCCGTTTTCCACATGGTAACGCAGCACGcatatgaatatcctccttag-3' \\
\hline 118 & $\mathrm{p} 49$ & 5'-GCACGCAACGCAAAATGAGTGGCGGCGGCAACTTGTTGACTCTGGAAAGAgtgtaggctggagctgcttc-3' & 5'-ATTATTATTATACCGAGTCGGGGATCAATAATTTGAGAAGCGTGTTCTGAcatatgaatatectcettag-3' \\
\hline 119 & odv-e18 & 5'-TAATAATATGATTTACACAGATCCCACTACTGGCGCTACGACTAGCACAGgtgtaggctggagctgettc-3' & 5'-TTTTATACACTTATCTACAACATTTGCCTTTGAGGCGTGTTCATAAAGGGcatatgaatatcctccttag-3' \\
\hline 120 & odv-ec27 & 5'-ATAAGTGTATAAAAATGAAACGTGTCAAATGCAACAAAGTTCGAACGGTCgtgtaggctggagctgcttc-3' & 5'-GAATTTATTTTGTCAAAATCTTCAATGGTAAATTTGAATTTGCTTAACAGcatatgaatatcctccttag-3' \\
\hline 121 & & 5'-ACATCAGGCTTTAATATATATAATTTTAATAAATAATTAAATAATATACAgtgtaggctggagctgcttc-3' & 5'-TAACAAGTTTCTATACATGCGAGCCGCACACCCGCTGCCCGTGCGATCGTcatatgaatatcctccttag-3' \\
\hline 122 & & 5'-GCAACTGAAACAATATTCAACATGAACGTCAATTTATACTGCCCTAATGGgtgtaggctggagctgcttc-3' & 5'-AGAGTGGCTTTCTAGTTGCACAACACTATTATCGATTTGCAGTTCGGGACcatatgaatatcetcettag-3' \\
\hline 123 & ie-1 & 5'-AGTTGCAAGTTGACATTGGCGGCGACACGATCGTGAACAACCAAACGACTgtgtaggctggagetgcttc-3' & 5'-TTTTTTTATATTTACAATTTAGTTTTTGTTCCGCAAACGTTATAGCGTCGcatatgaatatcctccttag-3' \\
\hline 124 & odv-e56 & 5'-TATATTTATTAAATAAGTTTGCTTTAAAATGAGTTTTTTTACAAATCTTCgtgtaggctggagctgcttc-3' & 5'-ATATTTTATATTATTTTTGTCTTTTATTATCGACGAGGGGCCGTTGTTGAcatatgaatatcctccttag-3' \\
\hline 125 & & 5'-ATTTATTGAAACGGTATATAACTTTAGCGATCATACAATGGAGAGATATCgtgtaggctggagctgcttc-3' & 5'-TTTAAAGCAAACTTATTTAATAAATATATCACAGTAAAGGTTTTGCAAAAcatatgaatatcctccttag-3' \\
\hline 126 & & 5'-AATAGTTTATTTGTTTTTTATAATTATTTTTATTGTTTTGAAATCTAAAAgtgtaggctggagctgcttc-3' & 5'-ATAAATTTTATTTTTATTTTTGTTTTGGTTAGCAGTACATCCATAATCTGATcatatgaatatcctccttag-3' \\
\hline 127 & ie-2 & 5'-GCACAGTTCAAGCCTCACAGCCTAACGAACAGTATCGCTACCAGCCAGCCgtgtaggctggagctgettc-3' & 5'-TATTTATATTATTTACAACACTTAAGGTTTAGACATCTCAATAGTGTATA catatgaatatcctccttag-3' \\
\hline 128 & pe38 & 5'-TTGTCAACTCGTAAGCACAGTTTGTTGCGGAGAGCCTGCCAATAAGCAAA gtgtaggctggagetgcttc-3' & 5'-TACATACATATCACATTAATTTTCAAACCCAAAATTAACAGTAACATTTAcatatgaatatcetcettag-3' \\
\hline 129 & & 5'-AACGTTTTTTATTTGTTTTTTTTATTTTATGTGATTAAGAAACTTTTAACgtgtaggctggagctgcttc-3' & 5'-ATTGTTATTTTAAATTATAATATCATTTAAATTTTTATGATGCAAGAATTcatatgaatatcctccttag-3' \\
\hline 130 & ptp & 5'-GAGTACATATTTAGTTACGTTTCTGAGATAAGATTGAAAGCACGTGTAAAgtgtaggetggagctgettc-3' & 5'-ATTAATAAATCTTGAACGTAATTTTGTCTTTCAATTTTGTGACCTCTGGCcatatgaatatcctccttag-3' \\
\hline 131 & bro-d & same as bro-a 5 ' primer & 5'-TTAATATTATTTGCATTTTTTAACAAATACTTTATTCTATTTTCAAATTGcatatgaatatcctccttag-3' \\
\hline 132 & bro-e & 5'-GTTTGTTTTGCGCTTTCGTCGAATCTCTACGCGTAGTTTGTAAATAAAAAgtgtaggetggagctgcttc-3' & 5'-TTACAACGACGCGTGACGATTCGACGAAAGCGCGAAACAAACTGAACGGAcatatgaatatcctcettag-3' \\
\hline 133 & & 5'-AATATATTATTGGATAAAGTTGCATTAAATGAAACTAACTTACAAGATGGgtgtaggetggagctgettc-3' & 5'-AACTGAGAACAACTAGTAGTGGTGTTGCTACAAATTCCCTCCGGCGTTGAcatatgaatatcctcettag-3' \\
\hline 134 & & 5'-TTAATGCAACTTTATCCAATAATATATTATGTATAGCACGTCAAAAATTAgtgtaggctggagctgcttc-3' & 5'-TCTTGCACCAGCGGTCCCGATTCGACTAACGGTCGATTGGATGGCTTAATcatatgaatatcctccttag-3' \\
\hline 135 & lef-2 & 5'-CGTGTGGAGTCCTCTCATTAGCGCGTCATGTTTAGACAAGAAAGCTACAT gtgtaggctggagctgettc-3' & 5'-TAATTACAAATAGGATTGAGGCCCTTGCAGTTGCCAGCAAACGGACAGAGcatatgaatatcctccttag-3' \\
\hline
\end{tabular}




\begin{tabular}{|c|c|c|}
\hline ORF No. & Gene name & type \\
\hline $1(8)$ & polyhedrin & $\mathrm{A}$ \\
\hline $2(9)$ & p78/83 & $\mathrm{C}$ \\
\hline $3(10)$ & Pk1 & $\mathrm{C}$ \\
\hline $4(11) *$ & & A \\
\hline $5(13)$ & & A \\
\hline $6(14) *$ & lef-1 & $\mathrm{C}$ \\
\hline 7 (15) & egt & $\mathrm{A}$ \\
\hline $7 \mathrm{a}(-) *$ & & $\mathrm{~A}$ \\
\hline $8(16)$ & bv/odv-e26 & A \\
\hline $9(17)$ & & $\mathrm{A}$ \\
\hline $10(18)$ & & $\mathrm{A}$ \\
\hline $11(19) *$ & & $\mathrm{~A}$ \\
\hline $12(20 / 21)$ & arif-1 & A \\
\hline $13(22)$ & pif-2 & $\mathrm{A}$ \\
\hline $14(23)$ & f protein & A \\
\hline $15(24)$ & pkip & $\mathrm{B}$ \\
\hline $16(25)$ & $\mathrm{dbp}$ & $\mathrm{C}$ \\
\hline $17(26) *$ & & $\mathrm{~B}$ \\
\hline $18(27)$ & iap1 & $\mathrm{A}$ \\
\hline $19(28)$ & lef-6 & A \\
\hline $20(29) *$ & & $\mathrm{~A}$ \\
\hline $21(30)$ & & $\mathrm{A}$ \\
\hline $22(-)$ & bro-a & $\mathrm{A}$ \\
\hline $23(31)$ & sod & $\mathrm{A}$ \\
\hline $24(32)$ & fgf & $\mathrm{A}$ \\
\hline $25(34) *$ & & $\mathrm{C}$ \\
\hline $26(35)$ & ubiquitin & $\mathrm{A}$ \\
\hline 27 (36) & $39 \mathrm{k}$ & $\mathrm{A}$ \\
\hline $28(37)$ & lef-11 & $\mathrm{D}$ \\
\hline $29(38)$ & nudix & $\mathrm{A}$ \\
\hline 30 (39) & $\mathrm{p} 43$ & A \\
\hline $31(40) *$ & p47 & $\mathrm{D}$ \\
\hline $32(41)$ & lef-12 & $\mathrm{A}$ \\
\hline $33(42)$ & gta & $\mathrm{A}$ \\
\hline $34(43)$ & & $\mathrm{A}$ \\
\hline $35(44) *$ & & $\mathrm{~A}$ \\
\hline $36(45) *$ & & $\mathrm{~A}$ \\
\hline $37(46)$ & odv-e66 & $\mathrm{A}$ \\
\hline $38(47) *$ & ets & A \\
\hline $39(50)$ & lef-8 & $\mathrm{D}$ \\
\hline $40(51) *$ & dnaJ domain & $\mathrm{C}$ \\
\hline
\end{tabular}




\begin{tabular}{|c|c|c|}
\hline ORF No. & Gene name & type \\
\hline $41(52)$ & & A \\
\hline $42(53)$ & & $\mathrm{C}$ \\
\hline 42a (53a) * & lef-10 & $\mathrm{D}$ \\
\hline $43(54)$ & vp1054 & $\mathrm{C}$ \\
\hline $44(55) *$ & & A \\
\hline $45(56) *$ & & A \\
\hline $46(57) *$ & & $\mathrm{~A}$ \\
\hline $47(58 / 59)$ & chaB-like & A \\
\hline $48(60) *$ & chaB-like & A \\
\hline $49(61)$ & fp & A \\
\hline $50(62) *$ & lef-9 & $\mathrm{D}$ \\
\hline $51(63) *$ & & A \\
\hline $52(64)$ & gp37 & A \\
\hline $53(65)$ & dnapol & $\mathrm{C}$ \\
\hline $54(66)$ & & $\mathrm{C}$ \\
\hline $55(67)$ & lef-3 & $\mathrm{D}$ \\
\hline $56(68)$ & & A \\
\hline 57 (69) & mtase & A \\
\hline $58(71)$ & iap2 & $\mathrm{A}$ \\
\hline 58a (72) * & & A \\
\hline $59(73)$ * & & $\mathrm{C}$ \\
\hline $60(74)$ & & A \\
\hline $61(75) *$ & & $\mathrm{C}$ \\
\hline $62(76)$ & & $C$ \\
\hline $63(77)$ & vlf-1 & $\mathrm{C}$ \\
\hline $64(78) *$ & & $\mathrm{C}$ \\
\hline $65(79) *$ & & B \\
\hline $66(80)$ & gp41 & $\mathrm{B}$ \\
\hline $67(81)$ & & $\mathrm{C}$ \\
\hline $68(82)$ & tlp & $\mathrm{A}$ \\
\hline $69(83) *$ & p95 & $\mathrm{C}$ \\
\hline $70(87) *$ & p15 & $\mathrm{A}$ \\
\hline $71(88)$ & $\operatorname{cg} 30$ & A \\
\hline $72(89) *$ & vp39 & $\mathrm{C}$ \\
\hline $73(90)$ & lef-4 & $\mathrm{D}$ \\
\hline 74 (91) & & $\mathrm{A}$ \\
\hline $75(92)$ & p33 & $\mathrm{C}$ \\
\hline $76(93) *$ & & $\mathrm{C}$ \\
\hline $77(94) *$ & odv-e25 & C \\
\hline 78 (95) & dnahel & $\mathrm{D}$ \\
\hline 79 (96) & pif-4 & C \\
\hline
\end{tabular}




\begin{tabular}{|c|c|c|}
\hline ORF No. & Gene name & type \\
\hline $80(-)$ & bro-b & A \\
\hline $81(-)$ & bro-c & A \\
\hline $82(98)$ & $38 \mathrm{k}$ & $\mathrm{C}$ \\
\hline 83 (99) & lef-5 & $\mathrm{D}$ \\
\hline $84(100)$ & p6.9 & $\mathrm{C}$ \\
\hline $85(101)$ & bv/odv-c42 & $\mathrm{C}$ \\
\hline $86(102) *$ & p12 & $\mathrm{C}$ \\
\hline 87 (103) & p45 & $\mathrm{C}$ \\
\hline $88(104)$ & vp80 & $\mathrm{C}$ \\
\hline $89(105)$ & he65 & $\mathrm{A}$ \\
\hline $90(106 / 107)$ & & $\mathrm{C}$ \\
\hline $91(108) *$ & & $\mathrm{~A}$ \\
\hline $92(109)$ & odv-ec43 & $\mathrm{C}$ \\
\hline 92a (110)* & & $\mathrm{A}$ \\
\hline $93(111) *$ & & $\mathrm{~A}$ \\
\hline 94 (114) & & $\mathrm{A}$ \\
\hline $95(115)$ & pif-3 & $\mathrm{A}$ \\
\hline 95a-96 (116-117) * & & $\mathrm{A}$ \\
\hline $96(117)$ & & $\mathrm{A}$ \\
\hline 97 (119) & pif-1 & $\mathrm{A}$ \\
\hline $98(120) *$ & & $\mathrm{~A}$ \\
\hline 98a (121)* & & $\mathrm{A}$ \\
\hline $99(122) *$ & & $\mathrm{~A}$ \\
\hline $100(123)$ & Pk2 & $\mathrm{A}$ \\
\hline $101(124) *$ & & $\mathrm{~A}$ \\
\hline $102(125)$ & lef-7 & $\mathrm{A}$ \\
\hline $103(126)$ & chitinase & $\mathrm{A}$ \\
\hline 104 (127) & v-cath & $\mathrm{A}$ \\
\hline $105(128)$ & gp64/67 & $\mathrm{C}$ \\
\hline $106(129)$ & p24 & $\mathrm{A}$ \\
\hline $107(130)$ & gp16 & $\mathrm{A}$ \\
\hline $108(131)$ & pp34 & $\mathrm{A}$ \\
\hline $109(132) *$ & & $\mathrm{C}$ \\
\hline $110(133)$ & an & $\mathrm{C}$ \\
\hline 110a (-) * & & $\mathrm{A}$ \\
\hline 111 (134) & & A \\
\hline $112(135)$ & p35 & $\mathrm{A}$ \\
\hline 113 (136) & p26 & A \\
\hline 114 (137) & p10 & A \\
\hline $115(138)$ & p74 & $\mathrm{A}$ \\
\hline $116(139)$ & me53 & $\mathrm{B}$ \\
\hline
\end{tabular}




\begin{tabular}{|c|c|c|}
\hline ORF No. & Gene name & type \\
\hline $117(141)$ & ie-0 & B \\
\hline $118(142)$ & p49 & C \\
\hline $119(143)$ & odv-e18 & B \\
\hline $120(144)$ & odv-ec27 & C \\
\hline $121(145)$ & & A \\
\hline $122(146)$ & & C \\
\hline $123(147)$ & ie-1 & D \\
\hline $124(148)$ & odv-e56 & A \\
\hline $125(149) *$ & & A \\
\hline $126(150)$ & & A \\
\hline $127(151)$ & ie-2 & A \\
\hline $128(153)$ & pe38 & A \\
\hline $129(154) *$ & & A \\
\hline $130(1)$ & ptp & A \\
\hline $131(2) *$ & bro-d & B \\
\hline $132(-)$ & bro-e & A \\
\hline $133(4) *$ & & A \\
\hline $134(5) *$ & & A \\
\hline $135(6)$ & lef-2 & C \\
\hline
\end{tabular}

\section{Table 2 Continued.}

